\title{
RELATION OF MICROWAVE EMISSION TO X-RAY EMISSION FROM SOLAR FLARES
}

\author{
TATSUO TAKAKURA \\ Dept. of Astronomy, University of Tokyo, Japan
}

\begin{abstract}
Recent observations of impulsive microwave and hard X-ray emissions during the early phase of the flares are briefly reviewed in order to deduce the dynamics of energetic electrons consistently from two view points of the microwaves and X-rays. An emphasis is put on the necessity of distinction between temporal and spatial variations so far confused in the interpretation of the time histories of the X-ray and radio emissions. The role of plasma turbulence on the dynamics of the energetic electrons is shown to be important in deducing the model of $\mathrm{X}$-ray and radio sources.
\end{abstract}

\section{Introduction}

The microwave emission and $\mathrm{X}$-ray emission from the flares are intimately related to each other although the wavelengths are quite far apart, because both emissions are caused by energetic electrons - either thermal or nonthermal - created in the flares. The characteristic and dynamics of such energetic electrons in and around the flare region have been deduced from the microwave and X-ray observations. Recent reviews on such a subject are given by Kane (1973a, 1974), Hudson (1973), De Feiter (1972) and Takakura $(1969,1973)$, and a new text book on the microwave bursts is given by Krüger (1972).

In order to investigate the characteristics of the energetic electrons, both microwave observation and X-ray observation have disadvantages and advantages. For example, the radio spectrum of gyro-synchrotron emission depends strongly on the magnetic field whose distribution in the corona is hard to estimate, while the X-ray spectrum of bremsstrahlung depends on the controversial target model, thin or thick. The ambiguities can be reduced if we refer to both X-ray and radio observations.

The aim of the present review is to show recent progress on the relation of microwave emission to X-ray emission mainly at the impulsive phase of the solar flares. A brief remark on the gradual thermal emissions from the sporadic hot coronal condensation is also given.

\section{Impulsive Emission}

(a) OBSERVATIONAL

At the early phase of the flares, impulsive emissions occur on microwaves and hard X-rays which are also accompanied by EUV flashes, kernals of $\mathrm{H} \alpha$ (Vorpahl, 1972) and sometimes flashes of $\mathrm{H}_{9}(\lambda 3835$, Zirin and Tanaka, 1973). The microwave burst is ascribed to the gyro-synchrotron emission and the $\mathrm{X}$-ray burst is ascribed to the collisional bremsstrahlung. Peterson and Winckler (1959) observed an impulsive hard $\mathrm{X}$-ray burst for the first time and noticed its association with impulsive microwave burst. A good correlation between them was shown by Kundu (1961), and it has now 
been confirmed by many observations (cf. review by Kane, 1974). Recently, Vorpahl (1972) has shown a better correlation between the peak radio flux at $8.8 \mathrm{GHz}$ and the total number of electrons above $100 \mathrm{keV}$ derived from the $\mathrm{X}$-ray measurements though mainly below $80 \mathrm{keV}$.

The time difference between the maxima of hard X-ray and microwave bursts has been statistically given by McKenzie (1972) to be $0.3 \pm 0.4 \mathrm{~min}$ using OSO- 3 X-ray data and the reported time of microwave bursts. Kane (1972) has shown with OGO-5

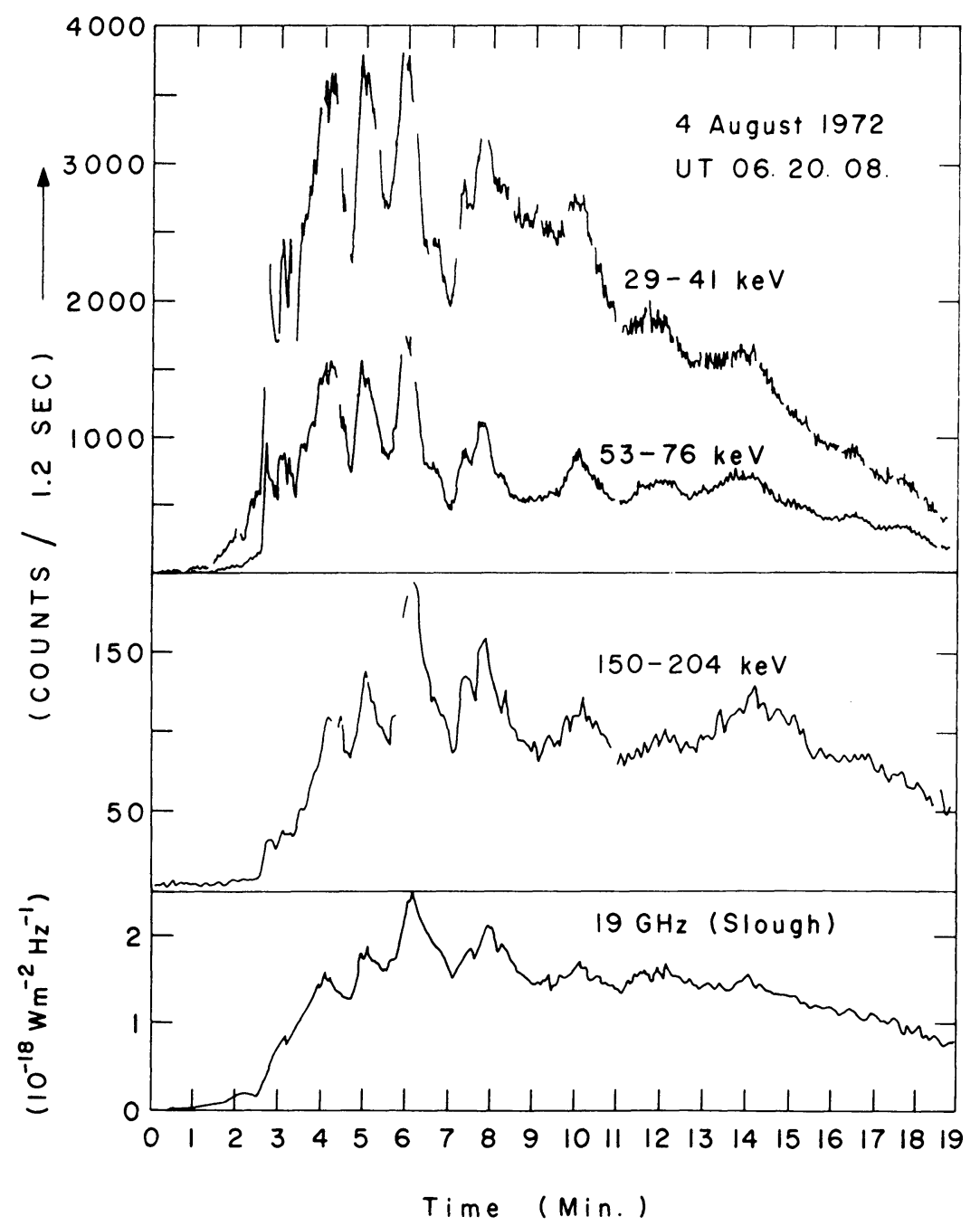

Fig. 1. X-ray and microwave bursts of 1972, August 4 during the flare of importance $3 B: 6^{\mathrm{h}} 20^{\mathrm{m}} 8^{\mathrm{s}}$ UT at $t=0$ on the time axis. The X-ray burst was observed with ESRO TD-1A satellite (Van Beek et al., 1973). The microwave burst was observed at Slough, England (Croom and Harris, 1973). The timing of this microwave burst reported was in error by about $40 \mathrm{~s}$. It has been corrected in the present figure in a comparison between the time profiles of $9.4 \mathrm{GHz}$ observed at Slough and Toyakawa. 
data that it is $\pm 6 \mathrm{~s}$ for small flares of importance 1 or less. Even for big flares, the time lag seems to be less than $10 \mathrm{~s}$ if we compare each correlated pulses as shown in Figure 1. The time difference, if any, is very important for the study of electron dynamics in the flare region. However the timing of microwave bursts so far recorded is generally not accurate to $\pm 6 \mathrm{~s}$ mainly due to low chart speed of the recorders.

The duration of microwave impulsive bursts ranges from 1 to $10 \mathrm{~min}$ in most cases when the time profile is simple and it is 2 to $30 \mathrm{~min}$ when the profile is complex. Great and prolonged bursts associated with intense flares have been designated as microwave type IV bursts, but we cannot find any distinctly different characteristics between the microwave impulsive bursts and the microwave type IV bursts from the microwave observations alone (Takakura, 1967). Their relation to hard X-ray bursts was thought to give a basis to divide the microwave bursts into subgroups, because the early measurements of hard X-rays showed that the hard X-ray bursts were only associated with the initial phase of the microwave bursts (Takakura, 1969). Therefore microwave bursts with longer duration are called 'the second phase'. Frost (1969) and Frost and Dennis (1971), however, showed two X-ray bursts having the second phase of longer duration. The other examples were obtained with the X-ray spectrometer on board the ESRO TD-1A (Hoyng et al., 1975, De Feiter, 1975). In August 1972, a series of large and small flares occurred in an active center and comprehensive data were collected at WDC, Boulder (Coffey, $1973 \mathrm{a}, \mathrm{b}$ ). Time profiles of the hard X-ray bursts given in the report by van Beek et al. (1973) are reproduced in Figure 1 together with a microwave burst at $19 \mathrm{GHz}$ given by Croom and Harris (1973). This radio burst is a typical great burst which may be called microwave type IV burst. It was associated with an importance $3 \mathrm{~B}$ flare on August 4. A correlation between time profiles of X-rays and radio waves is remarkable even in such a great complex event of long duration. It is thus hard to distinguish between the impulsive phase and the 2 nd phase, even if we refer to the time profile of hard X-rays. The burst seems to be composed of about 10 pulses. The individual peaks of $\mathrm{X}$-rays and microwaves coincide with each other within about $10 \mathrm{~s}$. The decay time of each radio pulse is slightly longer than that of X-rays. Generally, the correlation of time profiles of microwave burst with X-ray time profile seems best at about the frequency of maximum flux or at slightly higher frequencies, although the time profiles at the other frequencies are not shown in the figure. At the lower frequencies, the decay time becomes longer losing the similarity probably because of the optical thickness much greater than unity for the self absorption. The decay time of the microwave bursts at the frequency of maximum flux may correspond to the lifetime of electrons with effective energies of $100-300 \mathrm{keV}$, though the effective energy depends on the magnetic field, the size of the source and the number density of energetic electrons in the radio source. The effective energy increases with increasing frequency.

It is remarkable in Figure 1 that no appreciable difference in the duration of each pulse can be seen in the wide range of photon energies from 30 to $200 \mathrm{keV}$. Note that the duration of each pulse became longer accompanying the hardening of the spectrum at the later phase of the event. 
Another great event was observed on August 7 starting at about $15^{\mathrm{h}} 13^{\mathrm{m}}$ UT (Coffey, 1973a, b). This burst is also composed of several pulses and the correlation of time profiles of X-rays and microwaves ( 15.4 and $35 \mathrm{GHz}, \mathrm{AFCRL}$ ) is also good without any time difference greater than $10 \mathrm{~s}$. For a comparison, a typical impulsive event associated with a small flare with an importance - B on August 7 is shown in Figure 2.

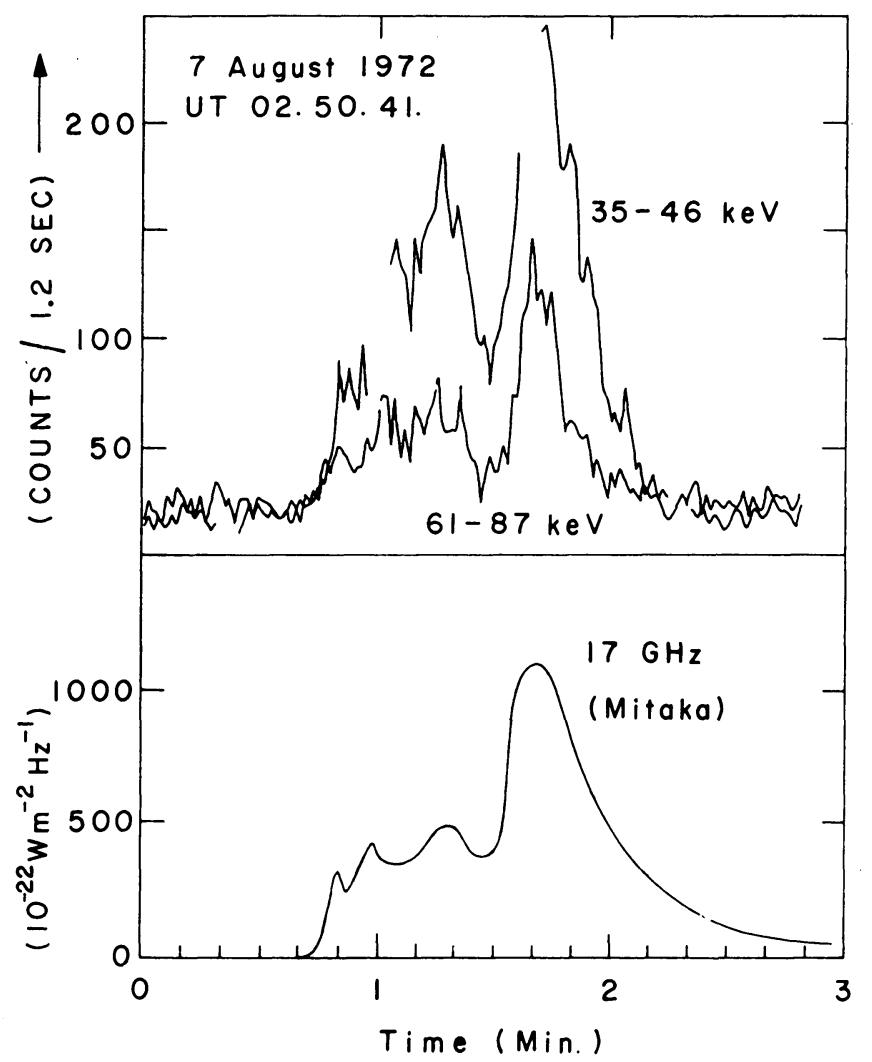

Fig. 2. X-ray and microwave bursts of 1972, August 7 during the subflare of importance $-B$. The X-ray burst was observed with ESRO TD-1A satellite (Van Beek et al., 1973).

In a comparison between this impulsive burst and a great burst shown in Figure 1, there seems no distinct difference between them except for the duration of each pulse and the whole duration of the burst.

Intense short lived pulses of $2 \mathrm{~s}$ or less were superposed on the hard X-ray bursts observed by Anderson and Mahoney (1974). The associated microwave bursts ( 3.75 and $9.4 \mathrm{GHz}$, by courtesy of Toyokawa) are similar in time profile but such short and intense pulses are not superposed on the main burst (except for a small pulse) even though the time constant of the radiometer is $1 \mathrm{~s}$.

Quasi-periodicity of the pulses has been reported (Parks and Winckler, 1969, 1971; Frost, 1969; Janssens and White, 1970; Janssens et al., 1973). 
In the analyses of the time history of microwave and X-ray bursts during a single flare, it is very important whether all pulses appearing in a single flare emitted from the same position or the individual pulses originate from different sources. The interpretations so far were implicitly based on the assumption that the X-ray source is only one during a single flare. Accordingly, the pulses on the time profile have been used to support the continuous injection model of electrons (e.g. Hudson, 1973), or they have been ascribed to the modulation of trapped electrons due to $\mathrm{MH}$ waves (Brown, 1973b) under the instantaneous injection model. The softening of hard $\mathrm{X}$-ray spectrum during a single flare has also been used to support the continuous injection model by Kane and Anderson (1970), while Brown (1972) has suggested that it may also be ascribed to an energy-dependent pitch angle distribution of electrons and nonuniform target density inside the X-ray source. However, if the individual pulses originate from different independent sources, quite different interpretation is required for the time history of microwave and X-ray bursts. That is, a time variation of the various characteristic parameters during a burst, such as spectral index, measured without spatial resolutions gives a mixture of spatial and temporal variations, from one source to another, rather than the time variation of energetic electrons in a single source. Note the variation of photon spectral index may also be ascribed to the temporal or spatial changes of a ratio between coronal (thin target) and chromospheric (thick target) components even though the spectral index of the electrons is constant. For example, when the thick target component is higher the photon spectral index becomes apparently smaller.

Time variations of source structure of microwave bursts have been measured with radio interferometers mainly at Toyokawa with one-directional beam-width of $1^{\prime}$. The radio observations have given that the radio source of intense microwave bursts is composed of several smaller sources showing time variations independent of each other (Enome, 1972; Enome and Tanaka, 1973). One example is shown in Figure 3. Four sources were resolved in this case. Only one hard X-ray burst source has so far been observed with some spatial resolution (Takakura et al., 1971). The spatial and time resolutions were not enough to resolve the source into smaller sources in that event. However, if we refer to the comparison made by Zirin and Tanaka (1973) between the optical flashes ( $\lambda$ 3835) and a hard X-ray burst (Figure 4 ) it is probable that the individual pulses in a single $X$-ray burst also originate from different sources. In this respect, hard X-ray observations with high spatial and time resolutions are highly desirable. The spatial resolution of the present microwave observations is also not high enough, although some high resolution measurements of one-directional source size were made (Hobbs et al., 1973; Kundu et al., 1974).

From the above consideration, it is highly probable that several independent sources are triggered successively during a single flare. The lifetime of individual source may be equal to the duration of each pulse appearing in the flux of X-ray and microwave bursts, e.g. about $1 \mathrm{~min}$ (full width at half maximum) in the case of Figure 1 and 15-20 s in case of Figure 2 for both X-ray and microwaves. In case of Figure 4, however, it is about $5 \mathrm{~s}$ for X-rays while $15-30 \mathrm{~s}$ for the microwaves. The difference of the 


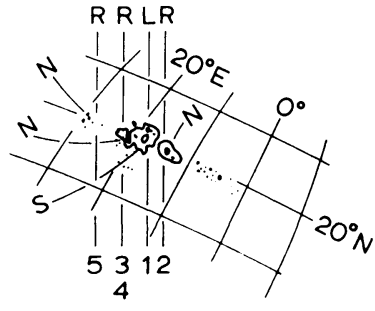

1969 MARCH 21

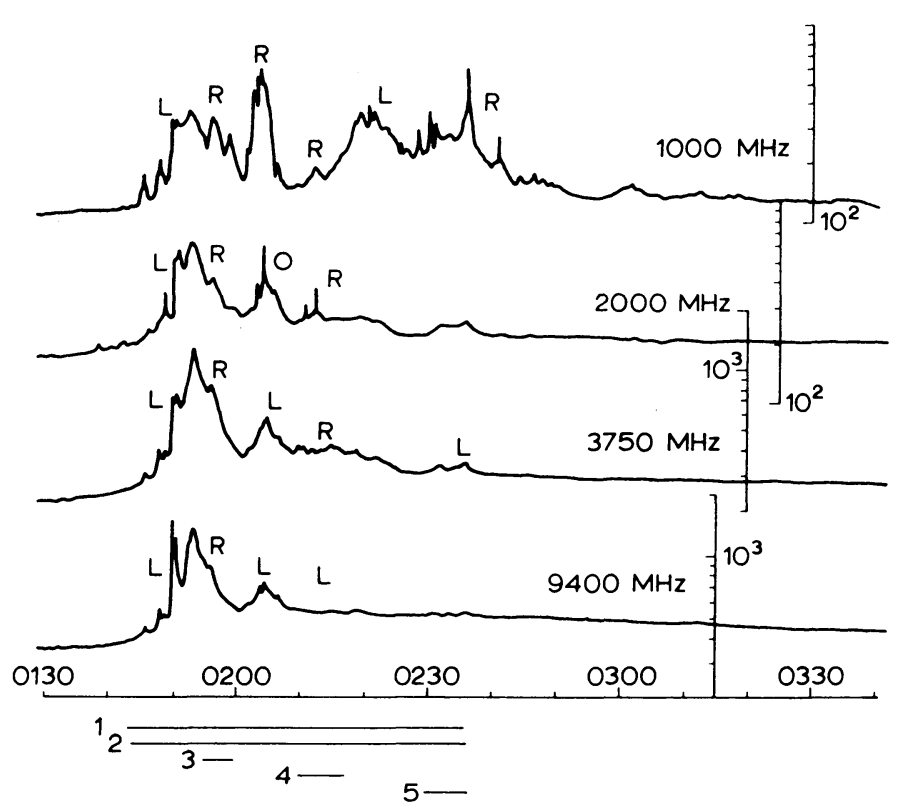

Fig 3. Time history of the microwave burst of 1969, March 21 and one-directional locations of radio sources at $3750 \mathrm{MHz}$ observed with the interferometer (Enomé, 1972; Enomé and Tanaka, 1973). The radio sources marked by 1 to 5 were detectable during the periods indicated by the same number on the time history. Individual sources showed independent time variation in intensity.

duration in this later case may indicate that their sources were not quite common and/or the lifetime of electrons with higher energies was longer.

(b) LIFETIME OF EACH PULSE

The problem to be discussed here is how to interpret such a short lifetime of each source as $1 \mathrm{~min}$ or less inferred from the observations.

As far as we can deduce from the August 2 event (Figure 4) the continuous injection model seems more reasonable as discussed by Zirin and Tanaka (1973). If we adopt such a thick target model, the duration of each pulse may merely correspond to the duration of a continuous acceleration of electrons in a magnetic flux tube. However, some hard X-ray bursts were associated with flares beyond the limb (cf. Takakura, 1973, Datlowe, 1975). It is a strong evidence that these X-ray bursts originated from the corona. There are also the other evidences favorable to as well as against the thin target model (Kane, 1974). Accordingly, at least in some events or during a certain period in a single event the coronal component of hard X-rays must be predominant. Then, if we adopt the thin target model, the decay of the burst is due to the energy loss of energetic electrons trapped in the magnetic flux tube and/or due to the escape of the electrons from the tube. Note that if the electrons escape mainly downwards into the chromosphere, the X-rays from the thick target cannot be ignored. 
Two kinds of energy loss have been considered so far. One is collision with thermal electrons and another is gyro-synchrotron radiation loss. Note that the $e$-folding decay time of number of electrons at a given energy is shorter by a factor $(\gamma-1)^{-1}$ than the $e$-folding decay time of energy of electrons if the power law electron spectrum with the index $\gamma$ is nearly maintained during the decay, and that the former corresponds

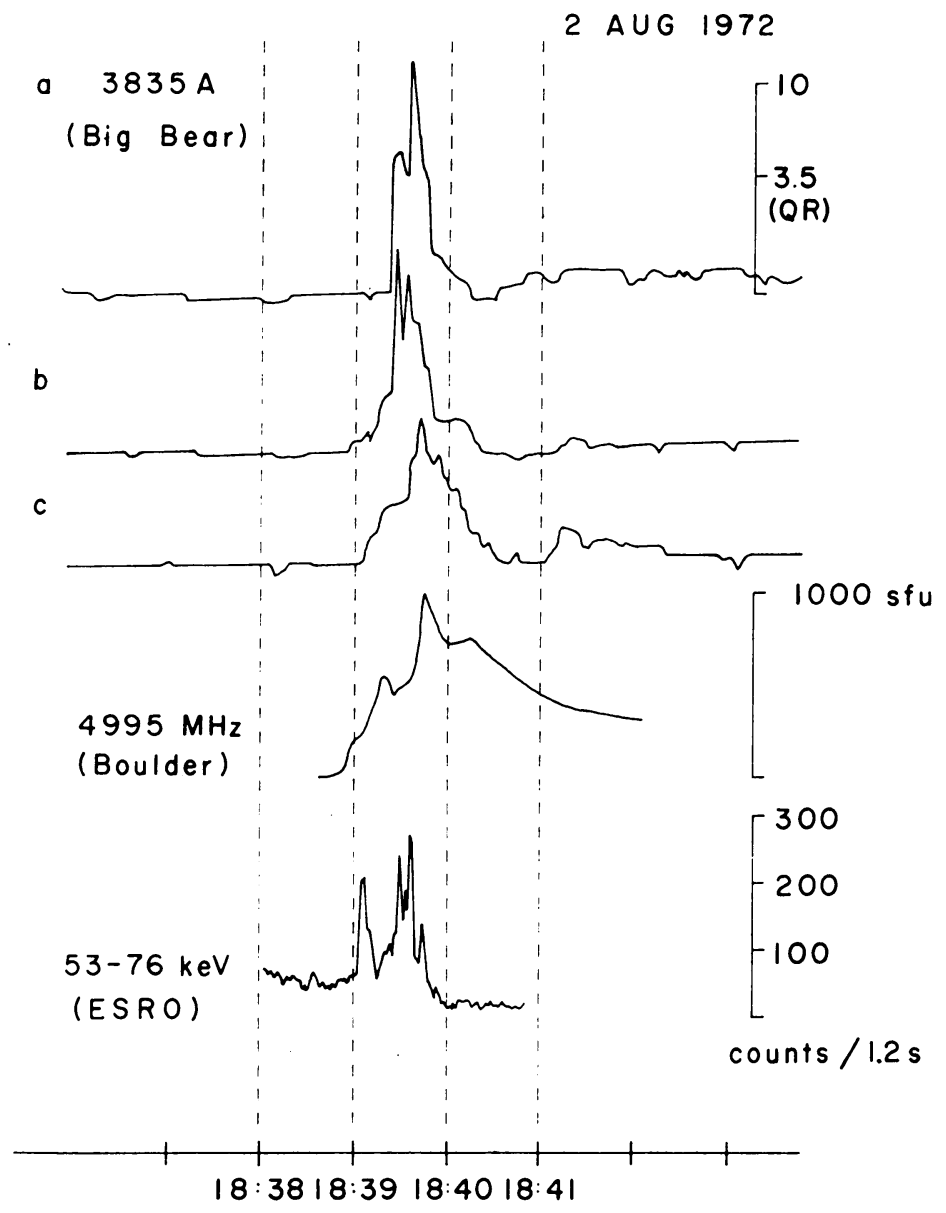

Fig. 4. Light curves of $\mathrm{H}_{9}(\lambda$ 3835) flashes measured at three positions, a, b and $\mathrm{c}$ at the flare of importance 1B of 1972, August 2 (Zirin and Tanaka, 1973). The lower two curves show microwave burst (Boulder) and X-rays (Van Beek et al., 1973). Note that the lifetime of individual pulses of X-rays is about $5 \mathrm{~s}$, while it is $15-30 \mathrm{~s}$ for microwaves.

to the decay time of fluxes of X-rays and microwave bursts. The $e$-folding decay time of microwave bursts at the frequency of maximum flux can be $30 \mathrm{~s}$ due to the collison loss if the effective number density of thermal electrons, $n_{\mathrm{e}}$, is $(2-8) \times 10^{9} \mathrm{~cm}^{-3}$ and $\gamma=4$ under the assumption that the effective energies of electrons emitting at the frequency of maximum flux are generally from 100 to $300 \mathrm{keV}$ in small flares, though 
they could be higher in larger flares. With increasing frequencies, however, the required number density $n_{\mathrm{e}}$ increases roughly in proportion to the frequencies as the effective electron energy increases. Gyro-synchrotron loss requires a magnetic field of about $3000 \mathrm{G}$ to give such a short decay time, even if $\gamma=4$. This field strength is too strong in the corona.

In this problem the role of plasma turbulence has not been considered except for a brief remark by Brown (1971). Strong ion-acoustic waves or electron plasma waves are most probably existing in the acceleration and trapping regions of electrons at the flares when a current density exceeds a critical value (Friedman and Hamberger, 1969; Takakura, 1971; Coppi and Friedland, 1971). In the presence of the turbulent ion-acoustic waves, the mean free path of energetic electrons due to the scattering by the waves is estimated to be several order smaller in the corona than that of classical Coulomb collisions. Note that the energy loss due to the scattering is comparatively small but can be larger than that of classical Coulomb collisions. It would be worthwhile to consider this effect in more detail, although we don't know whether it is essential in the present problem or not, because solar electrons travel through the interplanetary plasma exciting plasma waves as observed as type III radio bursts but as if there were no efficient interaction with the plasma in the scatter free events (Lin et al., 1973; Zaitsev et al., 1972). The evidence of the plasma turbulence occurring in the acceleration region and/or in microwave burst source during the early phase of the flares would be an associated dm wave burst in the frequency range of about $1000-300 \mathrm{MHz}\left(10^{10} \lesssim n_{\mathrm{e}} \lesssim 10^{9}\right)$, although the association of such the burst is rather infrequent.

Smith and Priest (1972) have given a rate of change in electron velocity $\mathbf{v}$ due to an interaction with isotropic plasma waves.

$$
\begin{aligned}
\left\langle\frac{\mathrm{d} v_{\mathrm{r}}}{\mathrm{d} t}\right\rangle & =-\frac{2(2 \pi)^{3} e^{2} c_{\mathrm{s}}^{2} \lambda_{\mathrm{e}}^{2}}{m_{\mathrm{e}}^{2} v^{4}} \int k^{3} W_{k} \mathrm{~d} k \simeq-\left(\begin{array}{c}
c_{\mathrm{s}} \\
v
\end{array}\right)^{2}\left\langle\begin{array}{c}
\mathrm{d} v_{\theta} \\
\mathrm{d} t
\end{array}\right\rangle \\
\left\langle\begin{array}{c}
\mathrm{d} v_{\theta} \\
-\mathrm{d} t
\end{array}\right\rangle & =\frac{2(2 \pi)^{3} e^{2} \lambda_{\mathrm{e}}^{2}}{m_{\mathrm{e}}^{2} v^{2}}\left(1+\frac{c_{\mathrm{s}}^{2}}{v^{2}}\right) \int k^{3} W_{k} \mathrm{~d} k
\end{aligned}
$$

where, $\left\langle\mathrm{d} v_{\mathrm{r}} / \mathrm{d} t\right\rangle$ and $\left\langle\mathrm{d} v_{\theta} / \mathrm{d} t\right\rangle$ are respectively the average rate of change of velocity in the direction of motion and in the direction perpendicular to $\mathbf{v}$,

$$
\begin{array}{ll}
c_{\mathrm{s}}=\left(\kappa T_{\mathrm{e}} / m_{\mathrm{i}}\right)^{1 / 2} & \text { is sound speed, } \\
\lambda_{\mathrm{e}}=\left(\kappa T_{\mathrm{e}} / m_{\mathrm{e}}\right)^{1 / 2} 2 \pi f_{\mathrm{p}, \mathrm{e}} & \text { is Debye length, }
\end{array}
$$

the suffixes $e$ and $i$ indicate electron and ion respectively, $T$ is temperature, $f_{\mathrm{p}}$ is plasma frequency and $W_{k}$ is an energy density of waves for a wave number $k$. For the isotropic turbulent ion-sound waves, they have given after Kadomtsev (1965)

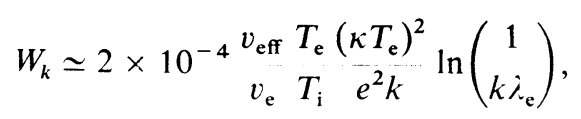


where $v_{\text {eff }}$ is the critical velocity of electrons for the onset of turbulent ion- sound waves and it depends on $T_{\mathrm{i}} / T_{\mathrm{e}}$ and $v_{\mathrm{e}}=\left(\kappa T_{\mathrm{e}} / m_{\mathrm{e}}\right)^{1 / 2}$ : we may set $v_{\mathrm{eff}} / v_{\mathrm{e}} \simeq T_{\mathrm{i}} / T_{\mathrm{e}}$. Equation (2) reduces to

$$
\left\langle\begin{array}{l}
\mathrm{d} v_{\theta} \\
\mathrm{d} t
\end{array}\right\rangle \simeq 0.71 \times 10^{-3} \pi^{4} f_{\mathrm{p}, \mathrm{e}} v_{\mathrm{e}}^{3} v^{-2} .
$$

A deflection time $\tau_{d}^{\mathrm{s}}$ due to the turbulent ion-sound waves may be given by

$$
\left\langle\begin{array}{c}
\mathrm{d} v_{\theta} \\
\mathrm{d} t
\end{array}\right\rangle=v / \tau_{\mathrm{d}}^{\mathrm{s}}
$$

Thus we have

$$
\tau_{\mathrm{d}}^{\mathrm{s}} \simeq{ }_{f_{\mathrm{p}, \mathrm{e}}}^{14}\left(\begin{array}{c}
v \\
v_{\mathrm{e}}
\end{array}\right)^{3} \simeq 1.8 \times 10^{8} n_{\mathrm{e}}^{-1 / 2}\left(E(\mathrm{keV}) / T_{\mathrm{e}}\right)^{3 / 2} \mathrm{~s},
$$

$n_{\mathrm{e}}$ in $\mathrm{cm}^{-3}, T_{\mathrm{e}}$ in degree and $E(\mathrm{keV})$ is the kinetic energy of an electron in $\mathrm{keV}$ in nonrelativistic range.

An energy exchange time $\tau_{\mathrm{t}}^{\mathrm{s}}$ due to the turbulent waves may be given by

$$
\frac{\mathrm{d} E}{\mathrm{~d} t}=m_{\mathrm{e}} v\left\langle\frac{\mathrm{d} v_{\mathrm{r}}}{\mathrm{d} t}\right\rangle=-\frac{E}{\tau_{\mathrm{t}}^{\mathrm{s}}} .
$$

Accordingly, we have

$$
\begin{aligned}
\tau_{\mathrm{t}}^{\mathrm{s}}= & -E\left\{m_{\mathrm{e}} v\left\langle\frac{\mathrm{d} v_{\mathrm{r}}}{\mathrm{d} t}\right\rangle\right\}^{-1} \simeq \frac{m_{\mathrm{i}}}{m_{\mathrm{e}}}\left(\frac{E}{\kappa T_{\mathrm{e}}}\right) \tau_{\mathrm{d}}^{\mathrm{s}} \simeq \\
& \simeq 3.7 \times 10^{18} n_{\mathrm{e}}^{-1 / 2}\left(E(\mathrm{keV}) / T_{\mathrm{e}}\right)^{5 / 2} \mathrm{~s}
\end{aligned}
$$

in the same units as used in Equation (6).

On the other hand, the deflection time $\tau_{\mathrm{d}}^{\mathrm{c}}$ and energy exchange time $\tau_{\mathrm{t}}^{\mathrm{c}}$ due to classical Coulomb collisions are

$$
\tau_{\mathrm{d}}^{\mathrm{c}} \simeq \tau_{\mathrm{t}}^{\mathrm{c}} \simeq 2.1 \times 10^{8} E(\mathrm{keV})^{3 / 2} / n_{\mathrm{e}} \mathrm{s}
$$

in the same units as used in Equation (6) for $m_{\mathrm{e}} c^{2} \gg E>4 \kappa T_{\mathrm{e}}$. Therefore,

and

$$
\alpha_{\mathrm{d}} \equiv \tau_{\mathrm{d}}^{\mathrm{s}} / \tau_{\mathrm{d}}^{\mathrm{c}} \simeq 0.85 n_{\mathrm{e}}^{1 / 2} T_{\mathrm{e}}^{-3 / 2}
$$

$$
\alpha_{\mathrm{t}} \equiv \tau_{\mathrm{t}}^{\mathrm{s}} / \tau_{\mathrm{t}}^{\mathrm{c}}={ }_{m_{\mathrm{e}}}^{m_{\mathrm{i}}} \frac{E}{\mathrm{e}} \alpha_{\mathrm{d}} \simeq 1.8 \times 10^{10} n_{\mathrm{e}}^{1 / 2} T_{\mathrm{e}}^{-5 / 2} E(\mathrm{keV})
$$

in the same units as used in Equation (6). For example, if $n_{\mathrm{e}}$ is $10^{9}$ to $10^{10} \mathrm{~cm}^{-3}$ and $T_{\mathrm{e}}$ is $10^{7}$ to $10^{6}$, the ratio $\alpha_{\mathrm{d}}$ ranges from $10^{-6}$ to $10^{-4}$, while incidentally $\alpha_{\mathrm{t}}$ is 0.02 to 20 for $10 \mathrm{keV}$ electrons. Accordingly, $10-100 \mathrm{keV}$ electrons cannot stream freely into the chromosphere as has been thought in the thick target model if the plasma turbulence is occurring on the way of the electrons to the chromosphere. 
A diffusion time $\tau_{\mathrm{D} \|}$ for the electrons to move a distance $l$ along the magnetic field may be given on the analogy of random walk, by

$$
\tau_{\mathrm{D} \|} \simeq \tau_{\mathrm{d}}^{\mathrm{s}}\left(l / v \tau_{\mathrm{d}}^{\mathrm{s}}\right)^{2}
$$

while the diffusion time $\tau_{\mathrm{D} \perp}$ perpendicular to the magnetic field may be given by

$$
\tau_{\mathrm{D} \perp} \simeq \tau_{\mathrm{d}}^{\mathrm{s}}\left(l / \varrho_{\mathrm{H}}\right)^{2},
$$

where $\varrho_{\mathrm{H}}=v / \omega_{\mathrm{H}, \mathrm{e}}$ is the gyro-radius of the electron. Substituting (6), we have

$$
\begin{aligned}
& \tau_{\mathrm{D} \|} \simeq 1.6 \times 10^{-27} l^{2} n_{\mathrm{e}}^{1 / 2} T_{\mathrm{e}}^{3 / 2} E(\mathrm{keV})^{-5 / 2} \mathrm{~s}, \\
& \tau_{\mathrm{D} \perp} \simeq 1.5 \times 10^{4}(H l)^{2} n_{\mathrm{e}}^{-1 / 2} T_{\mathrm{e}}^{-3 / 2} E(\mathrm{keV})^{1 / 2} \mathrm{~s},
\end{aligned}
$$

$n_{\mathrm{e}}$ in $\mathrm{cm}^{-3}, l$ in $\mathrm{cm}, E(\mathrm{keV})$ in $\mathrm{keV}$ and $H$ is magnetic field in Gauss. If we put $n_{\mathrm{e}}=10^{9} \mathrm{~cm}^{-3}, T_{\mathrm{e}}=10^{7}$ and $l=10^{9} \mathrm{~cm}$, the diffusion time along the lines of force for $100 \mathrm{keV}$ electrons is about $16 \mathrm{~s}$ and it decreases rapidly with increasing energy of electrons though the above equation is not valid in the relativistic energies. $\tau_{D \perp}$ is generally very long.

In conclusion, high energy electrons $(>100 \mathrm{keV})$, which contribute to the radio emission as the gyro-synchrotron process, may have such a short escaping time as $20 \mathrm{~s}$ or less if turbulent plasma waves are excited in and around the acceleration region of electrons. On the other hand, lower energy electrons cannot escape from the acceleration region in their lifetime given by the energy transfer time $\left(\tau_{t}^{\mathrm{s}}\right.$ or $\left.\tau_{\mathrm{t}}^{\mathrm{c}}\right)$. Furthermore, it seems difficult to have an unisotropic distribution of electron velocities required to have linear polarization and directivity of hard X-rays. The possible occurrence of such plasma turbulence in at least some events and/or during a limited period in a single flare may have caused many variations in the observed characteristics of hard X-ray and microwave impulsive bursts.

\section{(c) MODEL OF RADIO AND X-RAY SOURCES FOR IMPULSIVE PHASE}

In the first hard X-ray event, Peterson and Winckler (1959) made a rough comparison between the number of electrons producing hard X-rays in thick target and that producing microwave burst, finding a discrepancy of $10^{4}$. This discrepancy was, however, mainly based on their misunderstanding as pointed out by Takakura (1962), that they compared the time integrated number of electrons which produced the whole $\mathrm{X}$-rays in the thick target and the instantaneous number of electrons to emit the peak flux of microwaves. The discrepancy can be small if we adopt correctly the thick target model (Takakura, 1962; Hudson, 1972; Zirin and Tanaka, 1973).

Anderson and Winckler (1962) proposed a thin target model taking high target number density as $10^{12} \mathrm{~cm}^{-3}$, i.e., the chromosphere, and supposed that the microwave burst is also emitted at the same height in the magnetic field of $10^{3} \mathrm{G}$ from common electrons producing X-rays. This number density is too high for the thin target model and also for the radio source. If we take more reasonable value as $10^{10}-10^{9}$ $\mathrm{cm}^{-3}$ for the ion density under the thin target model and make more accurate esti- 
mate of electrons with power law distribution emitting microwaves in a uniform magnetic field of $500-1000 \mathrm{G}$, the discrepancy becomes again $10^{3}-10^{4}$ at the same energy range. Therefore Takakura and Kai (1966) proposed a model in which the sources of $\mathrm{X}$-rays and microwaves are not common.

Holt and Ramaty (1966), on the other hand, showed under the thin target model that the discrepancy can be reduced if we take into account the self absorption of gyro-synchrotron emission in rather weak magnetic field of 200-300 G together with a high energy cutoff of electrons. Further Takakura $(1972,1973)$ showed under the thin target model that the discrepancy can be negligible if we take into account two more factors, viz., the magnetic field in the radio source is nonuniform and the electron energy spectrum has steeper slope above $100 \mathrm{keV}$, though this latter factor is nearly equivalent to the high energy cutoff assumed by Holt and Ramaty. Following this model Anderson and Mahoney (1974) showed that X-ray and microwave bursts are consistently explained even for small flares.

Recently Kane (1973b, 1974) has proposed a combination model in which the hard $\mathrm{X}$-ray source extends from the lower chromosphere to the lower corona. The acceleration of electrons is continuous and a part of the accelerated electrons is mirrored in the magnetic field and the rest is scattered into the loss cone and precipitates into the lower chromosphere as Model 3 shown in Figure 5. In his model the mirrored electrons are escaping into the corona. However, another possibility is that most of the mirrored electrons are mirrored again at the other end of the magnetic flux tube to be trapped in the tube, even though the injection of electrons is continuous.

CORONA

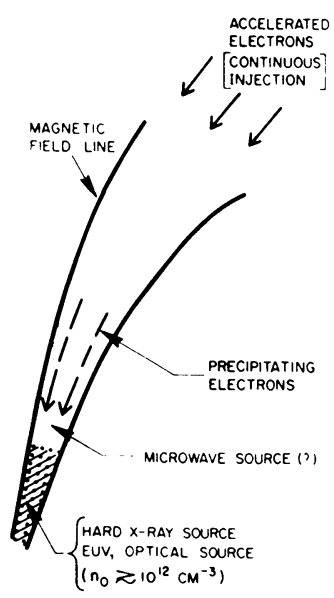

PHOTOSPHERE

MODEL - 1
CORONA

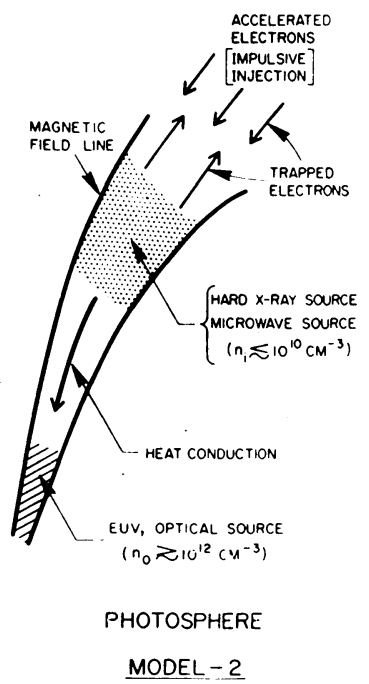

CORONA

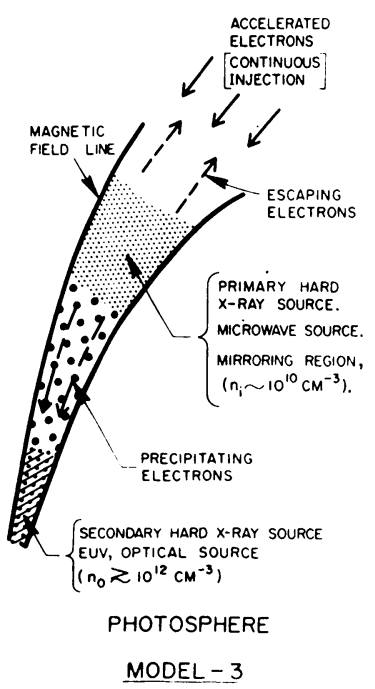

Fig. 5. Models of impulsive phase (Kane, 1974). Model 1. Continuous injection, thick target model (Syrovatskii and Shmeleva, 1972 and Hudson, 1972). - Model 2. Impulsive injection, thin target model (Takakura, 1973). - Model 3. Continuous injection, thin and thick targets model (Kane, 1973b). 
Recently Vorpahl and Takakura (1974) have suggested that the statistical result for the hard X-ray burst with shorter rise time to have harder photon spectrum could be ascribed to the existence of both chromospheric and coronal components; i.e., in cases where the chromospheric component predominates, the duration is shorter and the photon spectrum is harder and vice versa even though the electron spectrum index is almost constant from one event to another.

As already noted by Takakura (1973), it seems difficult in the present stage to say that the hard X-ray component from the chromosphere is less than that from the lower corona. The ratio between these two components probably varies with time and also spatially during a single flare and also from one flare to another. This would be one of the important problems to be solved by observations with high spatial and time resolutions. For example, very short and intense X-ray pulses as observed by Anderson and Mahoney (1974) and mentioned previously in subsection (a), would be emitted from the chromosphere since the corresponding microwave pulses were not observed.

Based on the interferometric observation at $35 \mathrm{GHz}$, Kawabata et al. (1973) tried to interpret the impulsive microwave burst by the gyro-radiation of $10-100 \mathrm{keV}$ electrons under the continuous injection thick target model. They have found, however, that their model cannot account for the observed less steep spectral slope of the radio burst at high frequencies (Kawabata, 1974, private communication). They are trying to modify the model including the reflecting electrons of higher energies above $100 \mathrm{keV}$. This model is thus similar to that suggested by Kane as shown in Figure 5.

A thermal model for the microwave impulsive burst has been proposed by Fürst (1973) in order to account for the short duration of impulsive microwave bursts. The burst is ascribed to the gyro-emission from thermal electrons with high temperature $\left(8 \times 10^{7}>T_{\mathrm{e}}>2 \times 10^{7}\right)$. The radio source is optically thick for the gyro-emission at any observed frequencies up to $15.4 \mathrm{GHz}$ due to the high temperature, and the short duration is attributed to the rapid cooling of the hot source due to heat conduction. Before we appreciate this model, however, comparison with associated soft- and hard-X-rays is required. A rough estimate shows that the expected soft X-rays at the peak of microwaves is more than one order higher than the observed flux. Another direct check is the measurement of brightness temperature of the radio source. Kundu et al. (1974) has given $1.2 \times 10^{9} \mathrm{~K}$ at $3.7 \mathrm{~cm}$ even for a very weak impulsive burst (18 sfu) observed with wide spacing interferometer. This result is against the thermal model.

\section{Gradual Emission}

There are small increases on microwaves showing gradual rise and fall (GRH burst). It may or may not be accompanied by an impulsive burst at its early phase. The correlation between the GRF burst and thermal soft X-rays is very good as shown by Hudson and Ohki (1972). Both are emitted due to a thermal process as shown by Kawabata (1960) from hot sporadic coronal condensation created at the flare. Based on a comparison between the X-rays and the microwaves, Hudson and Ohki (1972) 
have shown that the temperature of the condensation is effectively uniform. On the other hand Herring and Craig (1973) have proposed two temperature model, in which low temperature region has temperature of $(2-3) \times 10^{6} \mathrm{~K}$ and emission measure rises to $10^{51} \mathrm{~cm}^{-3}$ at the peak. If they have referred to the radio observation, however, this model could have been rejected as pointed out by Ohki (1974, private communication), since the low temperature region should emit the radio flux of 2 order higher than the observed value. Probably, the Skylab experiments have already given reliable informations about the structure of sporadic hot condensation.

In order to create coronal condensation excess gas should come from the chromosphere as pointed out by Hudson and Ohki (1972). The energy source to heat the chromosphere to cause expansion and supply the gas into the condensation is a controversial point. Hudson (1972) suggested that the chromospheric gas expands due to the heating by nonthermal electron streams emitting hard X-rays and Brown (1973a) has analysed this in more detail. However, the energy input into the hot condensation generally increases even after the decay of hard X-rays so that it is impossible for the nonthermal electrons to provide the heat input (Peterson et al., 1973, Datlowe, 1975). Another evidence is given by Neupert et al. (1974) that chromospheric EUV brightening has already reached $18 \%$ of its maximum when hard X-rays are just beginning to be emitted. Accordingly, Hirayama (1974) and Ohki (1973) has proposed a model that the gas in the chromosphere cause rapid expansion due to the heating by thermal conduction from the very hot $\left(T \gtrsim 2 \times 10^{7} \mathrm{~K}\right)$ coronal region in which the heating of gas is assumed to continue during the increasing phase of soft $\mathrm{X}$-rays, e.g., due to ohmic loss of current under the anomalous conductivity (Takakura, 1971). The front of heat flow propagates through the chromosphere as long as the energy flux exceeds the radiation loss thickening the transition region to emit excess EUV (Ohki, 1973). The speed of the front could be above $10 \mathrm{~km} \mathrm{~s}^{-1}$ at the layer with density of $10^{13} \mathrm{~cm}^{-3}$ if $T \gtrsim 2 \times 10^{7} \mathrm{~K}$ in the coronal heat source. Therefore, the flashes of EUV, $\mathrm{H} \alpha$ and $\mathrm{H}_{9}$ could also be ascribed to the heat conduction when the coronal temperature exceeds $10^{7.5} \mathrm{~K}$ (Takakura, 1973). Accordingly stationary state as Shmeleva and Syrovatskii (1973) has treated could not be applied during the early phase of flares.

\section{References}

Anderson, K. A. and Mahoney, W. A.: 1974, Solar Phys. 35, 419.

Anderson, K. A. and Winckler, J. R.: 1962, J. Geophys. Res. 67, 4103.

Brown, J. C.: 1971, Solar Phys. 18, 489.

Brown, J. C.: 1972, Solar Phys. 25, 158.

Brown, J. C.: 1973a, Solar Phys. 31, 143.

Brown, J. C.: 1973b, Solar Phys. 32, 227.

Coffey, H. E.: 1973a, Collected Data Reports on August 1972 Solar-Terrestrial Events, World Data Center A for Solar-Terrestrial Physics, Report UAG-28, Part I.

Coffey, H. E.: 1973b, Collected Data Reports on August 1972 Solar-Terrestrial Events, World Data Center A for Solar-Terrestrial Physics, Report UAG-28, Part II.

Coppi, B. and Friedland, A. B.: 1971, Astrophys. J. 169, 379.

Croom, D. L. and Harris, L. D. J.: 1973, see Coffey, 1973a, p. 210.

Datlowe D. W.: 1975, This volume, p. 191. 
De Feiter, L. D.: 1972, Space Sci. Rev. 13, 827.

De Feiter, L. D.: 1975, This volume, p. 283.

Enomé, S.: 1972, Thesis.

Enomé, S. and Tanaka, H.: 1973, in R. Ramaty and R. G. Stone (eds.), High-Energy Phenomena on the Sun, NASA SP-342, p. 78.

Friedman, M. and Hamberger, S. A.: 1969, Solar Phys. 8, 104.

Frost, K. J.: 1969, Astrophys. J. 158, L159.

Frost, K. J. and Dennis, B. R.: 1971, Astrophys. J. 165, 655.

Fürst, E.: 1973, Solar Phys. 28, 159.

Herring, J. R. H. and Craig, I. J. D. : 1973, Solar Phys. 28, 169.

Hirayama, T.: 1974, Solar Phys. 34, 323.

Hobbs, R. W., Jordan, S. D., and Webster, W. J.: 1973, Nature Phys. Sci. 243, 48.

Holt, S. S. and Ramaty, R.: 1969, Solar Phys. 8, 119.

Hoyng, P., Brown, J. C., Stevens, G., and Van Beek, H. F.: 1975, This volume, p. 233.

Hudson, H. S.: 1972, Solar Phys. 24, 414.

Hudson, H. S.: 1973, in R. Ramaty and R. G. Stone (eds.), High Energy Phenomena on the Sun, NASA SP-342, p. 207.

Hudson, H. S. and Ohki, K.: 1972, Solar Phys. 23, 155.

Janssens, T. J. and White, III, K. P.: 1970, Solar Phys. 11, 299.

Janssens, T. J., White, III, K. P., and Broussard, R. M.: 1973, Solar Phys. 31, 207.

Kadomtsev, B. B.: 1965, Plasma Turbulence, Academic Press, New York.

Kane, S. R.: 1972, Space Sci. Rev. 13, 822.

Kane, S. R. : 1973a, in R. Ramaty and R. G. Stone (eds.), High Energy Phenomena on the Sun, NASA SP-342, p. 55.

Kane, S. R.: 1973b, 3rd Meeting of the Solar Physics Division of AAS. Las Cruces, New Mexico.

Kane, S. R. : 1974, in G. Newkirk, Jr. (ed.), 'Coronal Disturbances' IAU Symp. 57, 105.

Kane, S. R. and Anderson, K. A.: 1970, Astrophys. J. 162, 1003.

Kawabata, K.: 1960, Rept. Ionos. Space Res. Japan 14, 405.

Kawabata, K.: 1974, Private communication.

Kawabata, K., Sofue, Y., Ogawa, H., and Omodaka, T.: 1973, Solar Phys. 31, 469.

Krüger, A.: 1972, Physics of Solar Continuum Radio Bursts, Akademie-Verlag, Berlin.

Kundu, M. R.: 1961, J. Geophys. Res. 66, 4308.

Kundu, M. R., Velusamy, T., and Becker, R. H.: 1974, Solar Phys. 34, 217.

Lin, R. P., Evans, L. G., and Fainberg, J.: 1973, Astrophys. Letters 14, 191.

McKenzie, D. L.: 1972, Astrophys. J. 175, 481.

Neupert, W. M., Thomas, R. J., and Chapman, R. D.: 1974, Solar Phys. 34, 349.

Ohki, K.: 1973, Thesis.

Ohki, K.: 1974, Private communication.

Parks, G. K. and Winckler, J. R.: 1969, Astrophys. J. 155, L117.

Parks, G. K. and Winckler, J. R.: 1971, Solar Phys. 16, 186.

Peterson, L. E., Datlowe, D. W., and McKenzie, D. L.: 1973, in R. Ramaty and R. G. Stone (eds.), High Energy Phenomena on the Sun, NASA SP-342, p. 132.

Peterson, L. E. and Winckler, J. R.: 1959, J. Geophys. Res. 64, 697.

Shmeleva, O. P. and Syrovatskii, S. I. : 1973, Solar Phys. 33, 341.

Smith, D. F. and Priest, E. R.: 1972, Astrophys. J. 176, 487.

Syrovatskii, S. I. and Shmeleva, O. P.: 1972, Sov. Astron. - A.J. 16, 273.

Takakura, T.: 1962, J. Phys. Soc. Japan 17, Suppl. A-II (International Conference on Cosmic Rays and the Earth Storm), p. 243.

Takakura, T.: 1967, Solar Phys. 1, 304.

Takakura, T.: 1969, Solar Flares and Space Research, North-Holland, p. 165.

Takakura, T.: 1971, Solar Phys. 19, 186.

Takakura, T.: 1972, Solar Phys. 26, 151.

Takakura, T.: 1973, in R. Ramaty and R. G. Stone (eds.), High Energy Phenomena on the Sun, NASA SP-342, p. 179.

Takakura, T. and Kai, K.: 1966, Publ. Astron. Soc. Japan 18, 57.

Takakura, T., Ohki, K., Shibuya, N., Fujii, M., Matsuoka, M., Miyamoto, S., Nishimura, J., Oda, M., Ogawara, Y., and Ota, S.: 1971, Solar Phys. 16, 454. 
Van Beek, H. F., Hoyng, P., and Stevens, G. A.: 1973, see Coffey, 1973b, p. 319.

Vorpahl, J. A.: 1972, Solar Phys. 26, 397.

Vorpahl, J. A. and Takakura, T. : 1974, Astrophys. J. 191, 563. Also in this volume, p. 237.

Zaitsev, V. V., Mityakov, N. A., and Rapoport, V. O.: 1972, Solar Phys. 24, 444.

Zirin, H. and Tanaka, K.: 1973, Solar Phys. 32, 173. 